\title{
Impaired Spatial Learning and Hyperactivity in Developing Rats Exposed to Intermittent Hypoxia
}

\author{
BARRY W. ROW, LEILA KHEIRANDISH, JENNIFER J. NEVILLE, AND DAVID GOZAL \\ Department of Pediatrics [B.W.R., L.K., J.J.N., D.G.], and Department of Pharmacology and Toxicology [D.G.], \\ Kosair Children's Hospital Research Institute, University of Louisville, Louisville, Kentucky 40202, U.S.A.
}

\begin{abstract}
ABS
Obstructive sleep apnea (OSA) is a frequent medical condi-
tion and is associated with cognitive impairments in adults and
with hyperactivity and decreased school performance in children.
In an adult rodent model, intermittent hypoxia (IH), such as
occurs in OSA, is associated with neurodegenerative changes in
the hippocampus and cortex and with spatial learning deficits.
Because a unique developmental window of neural vulnerability
to IH is present, we hypothesized that exposure to IH throughout
the vulnerable ages would result in increased behavioral impair-
ments in the juvenile rat. Rat pups were therefore exposed to
either room air or IH beginning at postnatal (PN) d 10 until PN
d 30 . Learning and memory were assessed via a standard place-
training version of the Morris water maze beginning at PN d 25.
Locomotor activity was assessed on PN d 29 and 30 . Pups
exposed to IH displayed significant spatial learning impairments,
\end{abstract}
ABSTRACT

and exposed male rats but not female rats displayed increased locomotor activity in the open field. Collectively, these findings indicate that exposure to $\mathrm{IH}$ at an age that corresponds to the peak incidence of OSA in children induces substantial learning impairment and gender-dependent behavioral hyperactivity in the juvenile rat. We postulate that this novel experimental model may allow for future exploration of mechanisms underlying the neurobehavioral deficits of children with OSA. (Pediatr Res 52: 449-453, 2002)
Abbreviations
OSA, obstructive sleep apnea
IH, intermittent hypoxia
RA, room air
$\mathbf{P N}$, postnatal

The clinical syndrome of OSA, a condition characterized by repeated episodes of upper airway obstruction during sleep, affects approximately $5 \%$ of the general population and approximately $2 \%$ of children $(1,2)$. Neurocognitive deficits, such as excessive daytime sleepiness, learning impairments, and an increased incidence of hyperactivity and aggressive behavior in children, particularly male children, comprise one of the primary morbidities associated with pediatric OSA (3-9). The IH and sleep fragmentation that are associated with OSA have both been proposed to contribute to the cognitive deficits found in OSA patients, necessitating the development of an appropriate animal model to explore the effects of $\mathrm{IH}$ on cognitive function (10-12). Work from our laboratory has recently demonstrated that exposure to $\mathrm{IH}$ during sleep does not induce substantial sleep disturbances, and is associated with impaired spatial learning in the adult rat, as well as with

Received June 7, 2001; accepted March 22, 2002.

Correspondence : David Gozal, M.D., Kosair Children's Hospital Research Institute, University of Louisville, Baxter Biomedical Research Building, Suite 321, 570 South Preston St., Louisville, KY 40202, U.S.A.; e-mail: david.gozal@louisville.edu

Supported by National Institutes of Health grants HL69932, HL63912, and HL66358; American Heart Association grant AHA- 0050442N; and The Commonwealth of Kentucky Research Challenge Trust Fund. B.W.R. is supported by F32 HD42395 from the National Institutes of Health.

DOI: 10.1203/01.PDR.0000030718.77598.A4 increased apoptosis in the cortex and CA1 region of the hippocampus (13).

It has become increasingly clear that the magnitude of neurobehavioral impairments in children with OSA is greater than that seen in adults for a comparable degree of sleep disordered breathing. In fact, while an apnea-hypopnea index of 20 events per hour of sleep is considered trivial in the adult patient, it represents a severe case of OSA in children. Furthermore, although rat pups $<5 \mathrm{~d}$ of age are more resistant to the apoptotic events induced by $\mathrm{IH}$ in the hippocampus and cortex, there is a subsequent period of maximal vulnerability to IH which occurs between PN d 10 and 25, an age that corresponds to the peak incidence of OSA in children (14). Such findings clearly indicate that, at least anatomically, there is a period of increased susceptibility to $\mathrm{IH}$ in the juvenile rat. However, the behavioral effects of $\mathrm{IH}$ in the juvenile rat are unknown. The present study was therefore conducted to determine whether exposure to IH would induce neurobehavioral deficits in the juvenile animal.

\section{METHODS}

The experimental protocols were approved by the Institutional Animal Care and Use Committee and are in close agreement with the National Institutes of Health guide for the 
care and use of laboratory animals. All efforts were made to minimize animal suffering, to reduce the number of animals used, and to use alternatives to in vivo techniques.

Animals. Six time-pregnant Sprague Dawley rats were obtained from a commercial breeder (Charles River Breeding Laboratories, Portage, MI, U.S.A.) and delivery times were recorded as PN d 0. Upon delivery, litters were culled to 12 pups and randomly assigned to one of the two experimental groups, i.e. RA or IH. Before weaning, the pups and their dams were housed together. Pups were group housed with littermates after weaning, which occurred at PN d 21. All animals were weighed and sexed at weaning. Animals were given unlimited access to laboratory chow and water, and were maintained on a 12-h light-dark cycle $(0600-1800 \mathrm{~h})$.

IH. Beginning at PN d 10, the pups were housed with their dams in four identical commercially designed chambers $(30 \times$ $20 \times 20$ in.; Oxycycler model A44X0, Reming Bioinstruments, Redfield, NY, U.S.A.) and exposed to either IH ( $n=$ $35)$ or RA $(n=35)$ for $14 \mathrm{~d}$ before behavioral testing. Gas was circulated around each of the chambers, attached tubing, and other units at $60 \mathrm{~L} / \mathrm{min}^{-1}$ (i.e. one complete change per $10 \mathrm{~s}$ ). The oxygen concentration was continuously measured by an oxygen analyzer, and was changed by a computerized system controlling the gas valve outlets, such that the moment-tomoment desired oxygen concentration of the chamber was programmed and adjusted automatically. Deviations from the desired oxygen concentration were met by addition of $\mathrm{N}_{2}$ or $\mathrm{O}_{2}$ through solenoid valves. Ambient $\mathrm{CO}_{2}$ in the chamber was periodically monitored and maintained at $<0.01 \%$ by adjusting overall basal ventilation. Humidity was measured and maintained at $40-50 \%$ by circulating the gas through a freezer and silica gel. Ambient temperature was maintained at $22-24^{\circ} \mathrm{C}$. The IH profile consisted of alternating RA and $10 \% \mathrm{O}_{2}$ every $90 \mathrm{~s}$, while the corresponding controls consisted of flushing RA into the chamber every $90 \mathrm{~s}$.

Morris water maze. The Morris water maze consisted of a white circular pool, $1.8 \mathrm{~m}$ in diameter and $0.6 \mathrm{~m}$ in height, filled to a level of $35 \mathrm{~cm}$ with water maintained at a temperature of $29^{\circ} \mathrm{C}(15,16)$. Pool water was made opaque by the addition of $150 \mathrm{~mL}$ of nontoxic white tempera paint. A Plexiglas escape platform (20 cm in diameter) was positioned $1 \mathrm{~cm}$ below the pool surface. Distinctive, geometric, extramaze cues were affixed to a white curtain surrounding the pool at specific locations and were visible to the rats while in the maze. Maze performance was recorded by a video camera suspended above the maze and interfaced with a video tracking system (HVS Imaging, Hampton, U.K.). Albino rats used in the experiments were temporarily tattooed with a black mark to allow video tracking.

Animals were handled daily by the experimenters before behavior testing starting at PN d 21. Animals were given an acclimation session consisting of a $30 \mathrm{~s}$ swim in the pool in the absence of the platform and spatial cues on PN d 24. Place learning was assessed over five daily training sessions starting at PN d 25. Each daily training session consisted of four training trials. Each rat was placed into the pool from quasirandom start points and allowed a maximum of $90 \mathrm{~s}$ to find the escape platform, where it remained for $15 \mathrm{~s}$. Rats that failed to locate the platform at the end of $90 \mathrm{~s}$ were manually guided to the platform. After completion of the trial, the rat was removed and placed in a separate holding cage until the start of the next trial. The position of the platform remained constant across trials. Trials were separated by $240 \mathrm{~s}$. Upon completion of the daily training session, rats were returned to their home cage. Performance during the training trials was assessed by the mean escape latencies and swim distances. Probe trials, in which the platform was removed to assess spatial bias, were conducted on $\mathrm{d} 3$ and at the completion of place training on $\mathrm{d}$ 6. All probes consisted of a 30-s trial with the platform removed and were conducted before any daily training session to remove any confounding effects of that day's training session. Time spent in the target platform location, relative and cumulative proximity to the previous platform location, and the number of target crossings over the previous location of the target platform were recorded and used as an indicator of spatial bias. After the probe trial, visual discrimination was assessed on three cued trials. Each rat was placed into the pool from quasi-random start points and allowed a maximum of $30 \mathrm{~s}$ to escape to a platform elevated $1 \mathrm{~cm}$ above the surface of the pool. Performance during the cued trials was assessed by the mean escape latencies and swim distances to locate the platform.

Open field. Locomotor activity was assessed in a black Plexiglas chamber $(22 \times 22 \times 22$ in.). Activity was recorded by a video camera suspended above the open field and interfaced with a video tracking system (Noldus, Sterling, VA, U.S.A.). Albino rats used in the experiments were temporarily tattooed with a colored mark to allow video tracking. On PN d 29 and 30, randomly selected animals from each group were acclimated to the open field for $10 \mathrm{~min}$ before the start of the activity monitoring session, which lasted for $10 \mathrm{~min}$. The chamber was thoroughly cleaned with $75 \%$ ethanol between trials to eliminate any confounding odor effects.

Data analyses. All data analyses were conducted with SPSS statistical software (SPSS, Chicago, IL, U.S.A.). Preliminary ANOVA revealed no significant difference between males and females in either condition in the water maze, therefore male and female groups were pooled for all water maze behavioral analyses. Two-way repeated measures ANOVA (treatment and trial block) were used to analyze performance during place and cue training. Separate one-way ANOVA were used to analyze probe trial performance and locomotor activity. Statistically significant $(p<0.05)$ main effects and interactions were post-tested with Tukey's HSD (honestly significant differences) and Student Newman-Keuls tests, as appropriate.

\section{RESULTS}

Body weight. Body weights were measured on PN d 24, before behavioral testing. One-way ANOVA revealed that rat pups exposed to IH had significantly lower body weights than age-matched controls housed in RA, $\left(\mathrm{F}_{3,660}=11.819, p<\right.$ 0.0001). Posthoc Student-Newman-Keuls analyses revealed that, within each treatment, no significant effects of gender on body weight occurred (Fig. 1).

Morris water maze. No significant differences occurred between male and female rats, and the data were therefore 


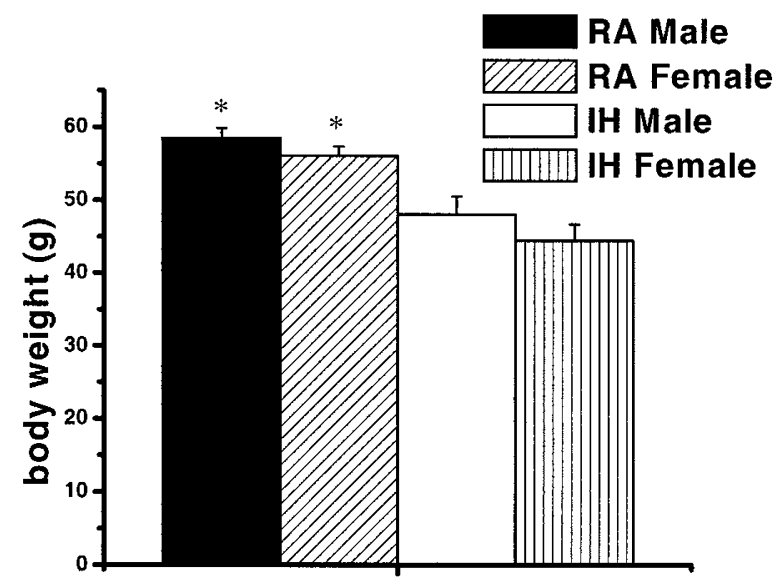

Figure 1. Mean body weight (in grams) of male ( $n=16$ per group) and female ( $n=19$ per group) rat pups exposed to $14 \mathrm{~d}$ of IH or RA. Bars represent standard errors. * IH $v s$ control, $p<0.001$, ANOVA.

pooled for subsequent analyses. Pups exposed to IH for $14 \mathrm{~d}$ ( $n$ $=35$ ) were significantly impaired in their spatial task acquisition as evidenced by both their escape latencies and swim path lengths (Fig. 2). ANOVA revealed a significant effect of $\mathrm{IH}$ on both escape latency $\left(\mathrm{F}_{1,68}=18.8, p<0.001\right)$ and path length $\left(\mathrm{F}_{1,68}=14.71, p<0.001\right)$. There were significant block effects for both escape latencies $\left(\mathrm{F}_{1,68}=221.18, p<0.0001\right)$ and path lengths $\left(\mathrm{F}_{1,68}=166.2, p<0.0001\right)$, indicating that the performance of all groups improved across trials. No
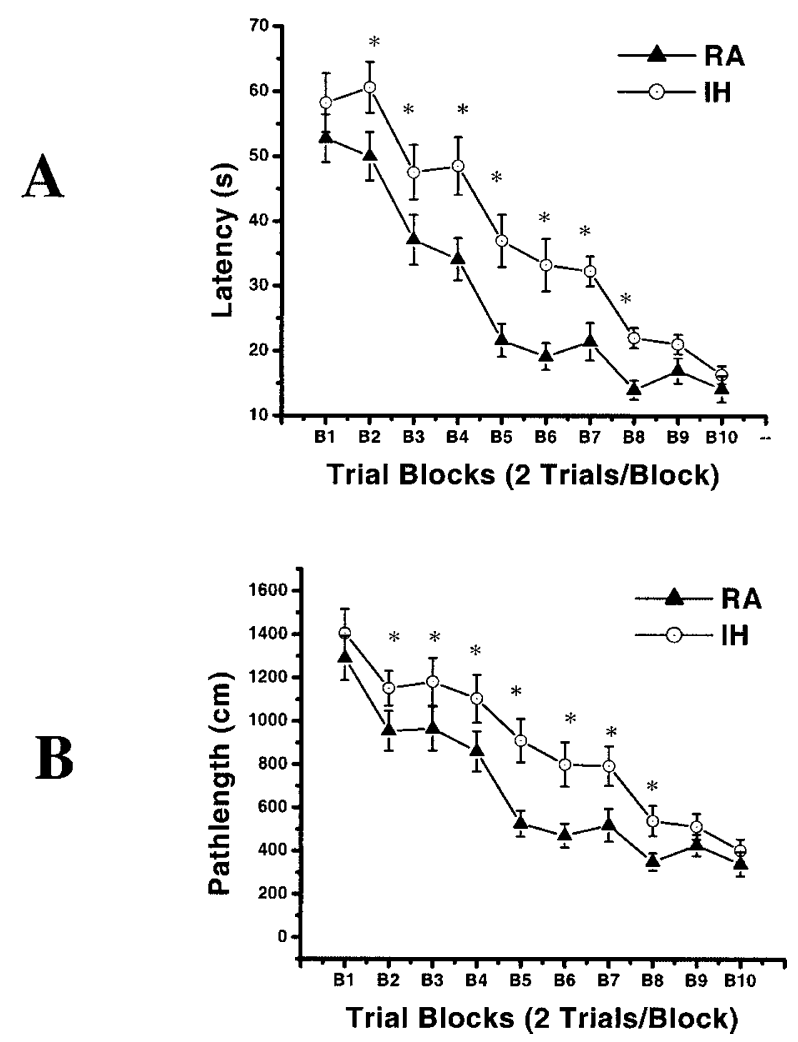

Figure 2. $(A)$ Mean escape latencies (in seconds) to locate the target platform during place training in rat pups exposed $14 \mathrm{~d}$ of $\mathrm{IH}(n=35)$ or RA $(n=35)$. $(B)$ Mean swim distances (in centimeters) to locate the target platform during place training in rat pups exposed to $14 \mathrm{~d}$ of $\mathrm{IH}(n=35)$ or RA $(n=35)$. Bars represent standard errors. ${ }^{*} \mathrm{IH} v s$ control, $p<0.01$, ANOVA. significant differences occurred between groups on the first block of $\mathrm{d} 1$, thereby confirming that all animals were naive to the platform location. IH animals were much more active while on the escape platform, and displayed a tendency to jump off the platform during the initial training sessions. Indeed, IHexposed pups were more likely to "jump" from the platform before being removed (46\%) compared with normoxic rats (28.7\%). This jumping behavior was not observed in any of the groups on subsequent training sessions.

During probe trials, IH-exposed pups showed substantial impairments in spatial memory compared with controls (Fig. 3 ). RA animals spent a greater percentage of time swimming in the target platform position $\left(\mathrm{F}_{1,68}=10.32, p<0.001\right.$ and $\mathrm{F}_{1,68}$ $=4.971, p<0.029$ for probe sessions 1 and 2, respectively). Analysis of the platform search patterns revealed that RA animals remained in closer proximity to the platform location $\left(\mathrm{F}_{1,68}=12.848, p<0.001\right.$ and $\mathrm{F}_{1,68}=5.837, p<0.018$ for probe sessions 1 and 2, respectively). RA animals also crossed the previous platform position significantly more times $\left(\mathrm{F}_{1,68}\right.$ $=12.585, p<0.001, \mathrm{~F}_{1,68}=5.064, p<0.029$ for probe sessions 1 and 2 , respectively).

In contrast, the performance of animals exposed to RA or IH was similar on the cued-platform task (Fig. 4, $p=\mathrm{NS}$ ), indicating that swimming motivation and ability were similar, and that the observed differences in spatial performance were not due to sensorimotor disturbances.

Open field. Mean locomotor activity in the open field, assessed as the distance covered during the trial times by male and female rat pups exposed to either RA or IH, is shown in Figure 5. ANOVA revealed a significant effect of $\mathrm{IH}$ on mean activity in the open field $\left(\mathrm{F}_{3,28}=3.52, p<0.028\right)$. Posthoc analyses revealed no significant locomotor activity differences for control male and female rats. However, male IH-exposed rats had higher locomotor activity in the open field $(p<0.05)$.

\section{DISCUSSION}

Our study unequivocally shows that an IH exposure during a critical period of neuronal vulnerability leads to substantial deficits in spatial learning. Moreover, increased locomotor activity in an open field occurs in IH-exposed male rats, and is uniquely reminiscent of the male preponderance that is found among children with attention deficit hyperactivity disorder (ADHD).

Before we discuss our findings, some methodological issues deserve comment. The IH protocol used in this study was developed to elicit arterial $\mathrm{Po}_{2}$ changes that would mimic the duration and magnitude of oxyhemoglobin desaturations occurring during obstructive apneic events. These preliminary studies showed that the nadir saturation achieved during the hypoxic component of the exposure was approximately $82 \%$ in juvenile rats (data not shown). Because sleep patterns in juvenile rats include both diurnal and nocturnal sleep epochs (17), $\mathrm{IH}$ exposures were applied throughout the 24-h period, rather than just during light hours. Thus, the findings reported herein may represent an overestimate of the neurobehavioral deficits that would occur if IH had been restricted to sleep periods only. 

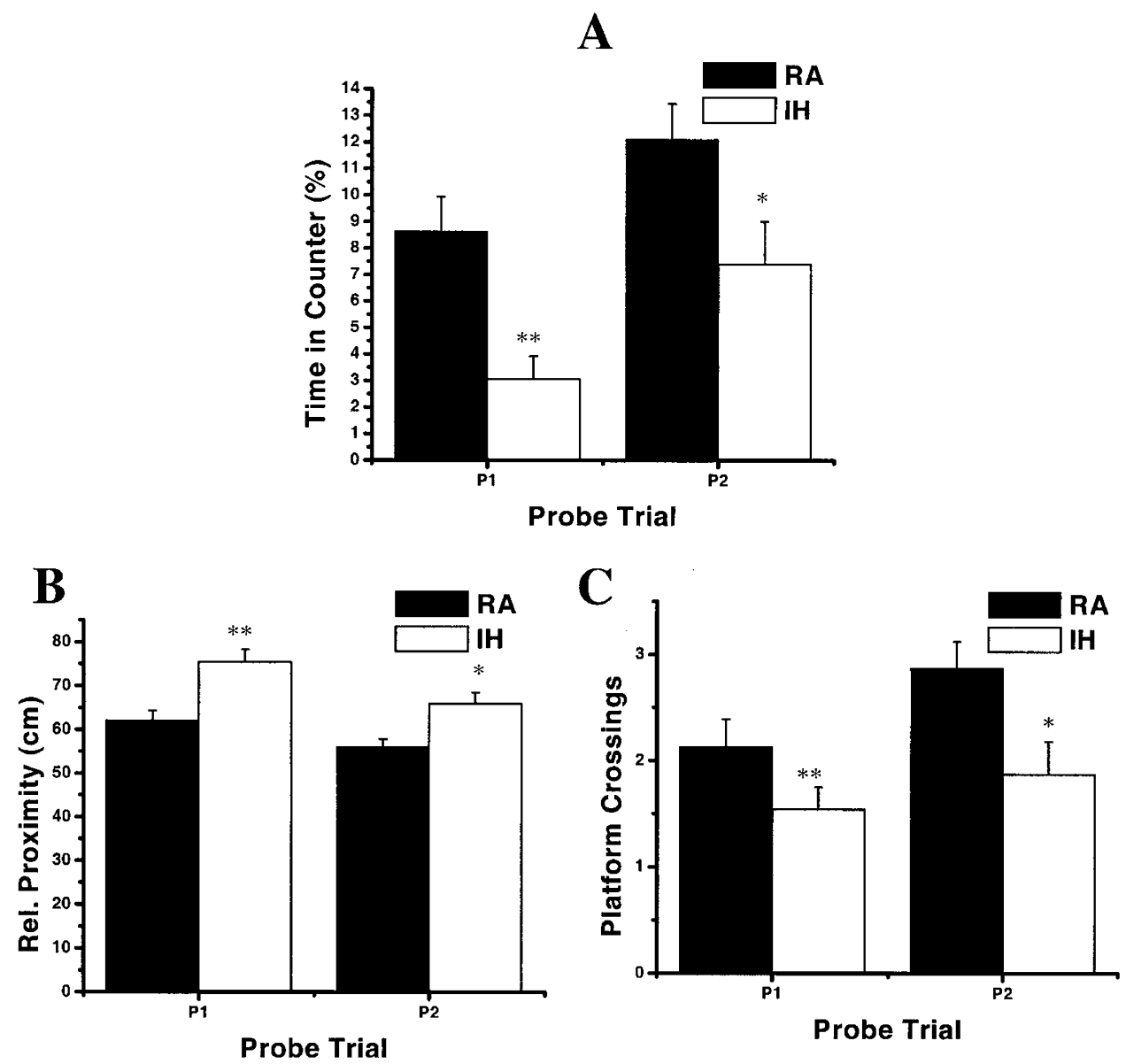

Figure 3. Probe trial performance of rat pups exposed to $14 \mathrm{~d}$ of $\mathrm{IH}(n=35)$ or RA $(n=35)$. Probe trial 1 was administered on $\mathrm{d} 3$ of behavioral testing and probe trial 2 was administered on $\mathrm{d} 6$ of behavioral testing. $(A)$ Mean percentage of time spent in target platform counter. $(B)$ Mean relative proximity to the target platform location. $(C)$ Mean number of platform position crossings. Bars represent standard errors. ${ }^{*} \mathrm{IH} v s$ control, $p<0.05$, ANOVA; ${ }^{* *} \mathrm{IH} v s$ control, $p<0.01$, ANOVA.

We are unaware of any substantive research addressing the effect of IH on neural development. Recent work from our laboratory has clearly established that intermittent hypoxic events during the sleep cycle of adult rats are associated with significant spatial learning deficits, and that such deficits occur in the absence of significant sleep alterations (13). Moreover, the neurobehavioral consequences of $\mathrm{IH}$ are preceded by marked increases in apoptosis in the cortex and CA1 region of the hippocampus, peaking at $48 \mathrm{~h}$ of IH exposure (13). Furthermore, developing rats at postnatal ages 10-25 d exhibit significantly more extensive apoptosis in these neural regions when exposed to $48 \mathrm{~h}$ of IH (14), indicating a developmentally regulated period of enhanced susceptibility to the episodic hypoxia that characteristically accompanies OSA. These findings are compatible with the concept of a slowly evolving or weak neural excitotoxicity that may occur as a consequence of impaired cellular energy metabolism, free radical production, and/or modifications in glutamate ion/receptor complexes (1820 ), and may lead to neuronal apoptosis (21). Our findings now extend from such anatomical studies, and clearly demonstrate that IH induced substantial spatial learning deficits in developing rats, and that these effects are gender independent. It remains unclear whether these effects are completely reversible after cessation of IH exposures or whether long-term deficits occur. This issue is of importance in light of two recent lines of evidence, namely that treatment of OSA in a large group of children academically failing during first grade leads to improved grades after treatment of sleep disorders (3), but also that 13-14-y-old children who snored between the ages of 2 and $6 \mathrm{y}$ were approximately three times more likely to be among those with lesser academic performances (4).

The increased locomotor activity found among male IHexposed rats is intriguing and establishes a potential causative link between the episodic hypoxia of pediatric OSA and symptoms of hyperactivity. Our findings are strikingly similar to those preliminarily reported by Decker et al. (22), who exposed rat pups to hypoxia between 7 and $10 \mathrm{~d}$ of age for $6 \mathrm{~h}$ and found increased locomotor activity in hypoxia-exposed rats. These findings are analogous to several studies that have documented that children with sleep disorders tend to have behavioral problems similar to those observed in children with ADHD. For example, a survey study of 782 children documented daytime sleepiness, hyperactivity, and aggressive behavior in children who snored, with $27 \%$ and $38 \%$ of children at high risk for a sleep or breathing disorder displaying clinically significant levels of inattention and hyperactive behavior, respectively (23). Similar studies reporting on smaller patient cohorts with proven OSA have also documented parental 

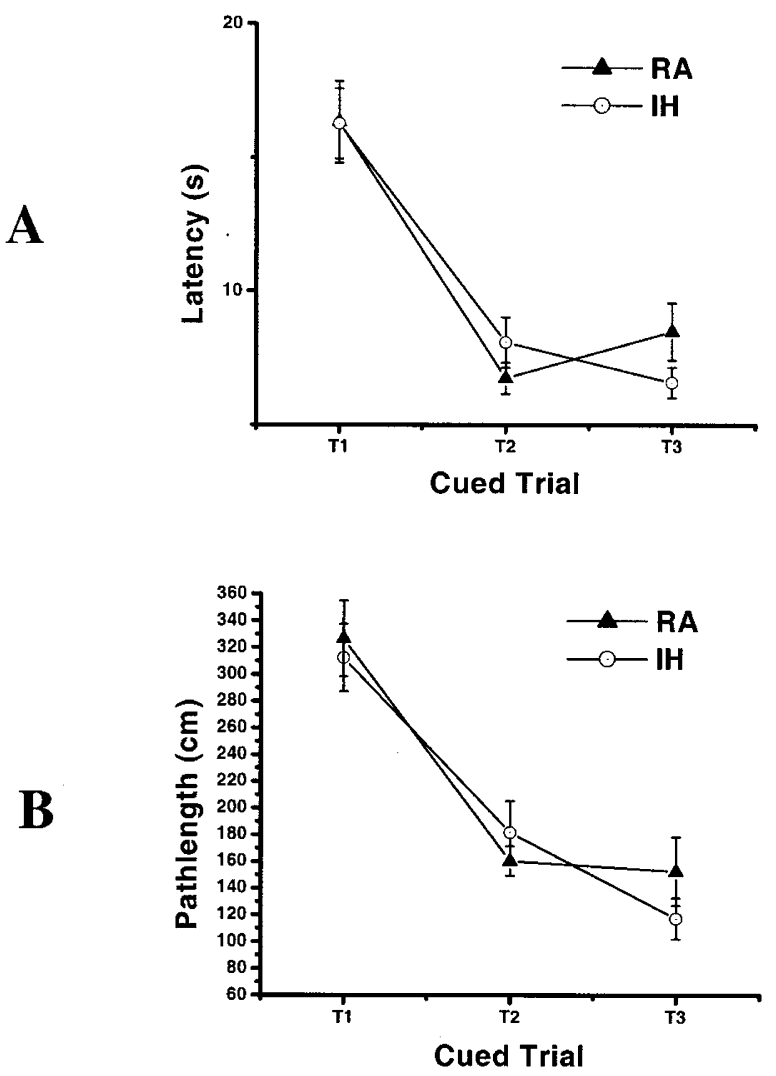

Figure 4. $(A)$ Mean escape latencies (in seconds) to locate the target platform during cued training in rat pups exposed $14 \mathrm{~d}$ of IH $(n=35)$ or RA $(n=35)$. $(B)$ Mean swim distances (in centimeters) to locate the target platform during cued training in rat pups exposed $14 \mathrm{~d}$ of IH $(n=35)$ or RA $(n=35)$.

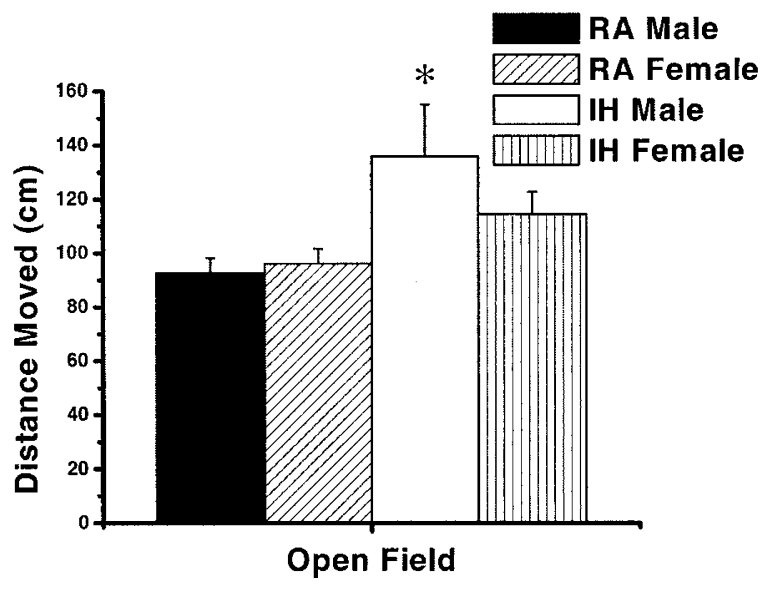

Figure 5. Locomotor activity in rat pups exposed to $14 \mathrm{~d}$ of IH or RA. Mean distance moved (in centimeters) of male $(n=8)$ and female $(n=8)$ pups exposed $14 \mathrm{~d}$ of IH and male $(n=8)$ and female $(n=8)$ pups exposed to RA. Bars represent standard errors. "IH $v s$ control, $p<0.05$, ANOVA.

reports of behavioral disturbances, and their improvement after treatment of OSA $(6,24,25)$. Conversely, children with ADHD are more likely to have sleep-disordered breathing, manifesting either as snoring (26) or as frank OSA (27).
The present study was not designed to elucidate the mechanisms underlying the neurocognitive deficits and behavioral phenotype resulting from IH exposures. However, it undoubtedly will provide a robust and useful animal model that should be of great value in gaining further insights into the role of episodic hypoxia in OSA-induced learning and behavioral morbidity, and their treatment.

\section{REFERENCES}

1. 1996 Sleep apnea: is your patient at risk? National Heart, Lung, and Blood Institute Working Group on Sleep Apnea. Am Fam Physician 53:247-253

2. Carroll JL, Loughlin GM 1995 Obstructive sleep apnea in infants and children: diagnosis and management. In: Kryger FA (ed) Principles and Practice of Sleep Meeting in the Child. WB Saunders Company, Philadelphia, pp 193-216

3. Gozal D 1998 Sleep-disordered breathing and school performance in children. Pediatrics 102:616-620

4. Gozal D, Pope Jr DW 2001 Snoring during early childhood and academic performance at ages thirteen to fourteen years. Pediatrics 107:1394-1399

5. Roehrs T, Merrion M, Pedrosi B, Stepanski E, Zorick F, Roth T 1995 Neuropsychological function in obstructive sleep apnea syndrome (OSAS) compared to chronic obstructive pulmonary disease (COPD). Sleep 18:382-388

6. Stradling JR, Thomas G, Warley AR, Williams P, Freeland A 1990 Effect of adenotonsillectomy on nocturnal hypoxaemia, sleep disturbance, and symptoms in snoring children. Lancet 335:249-253

7. Swift AC 1988 Upper airway obstruction, sleep disturbance and adenotonsillectomy in children. J Laryngol Otol 102:419-422

8. Guilleminault C, Korobkin R, Winkle R 1981 A review of 50 children with obstructive sleep apnea syndrome. Lung 159:275-287

9. Kales A, Caldwell AB, Cadieux RJ, Vela-Bueno A, Ruch LG, Mayes SD 1985 Severe obstructive sleep apnea-II: Associated psychopathology and psychosocial consequences. J Chronic Dis 38:427-434

10. Berry DT, Webb WB, Block AJ, Bauer RM, Switzer DA 1986 Nocturnal hypoxia and neuropsychological variables. J Clin Exp Neuropsychol 8:229-238

11. Bedard MA, Montplaisir J, Richer F, Rouleau I, Malo J 1991 Obstructive sleep apnea syndrome: pathogenesis of neuropsychological deficits. J Clin Exp Neuropsychol 13:950-964

12. Morisson F, Lavigne G, Petit D, Nielsen T, Malo J, Montplaisir J 1998 Spectral analysis of wakefulness and REM sleep EEG in patients with sleep apnoea syndrome. Eur Respir J 11:1135-1140

13. Gozal D, Daniel JM, Dohanich GP 2001 Behavioral and anatomical correlates of chronic episodic hypoxia during sleep in the rat. J Neurosci 21:2442-2450

14. Gozal E, Row BW, Schurr A, Gozal D 2001 Developmental differences in cortical and hippocampal vulnerability to intermittent hypoxia in the rat. Neurosci Lett 305:197-201

15. Morris R 1984 Developments of a water-maze procedure for studying spatial learning in the rat. J Neurosci Methods 11:47-60

16. Brown RW, Kraemer PJ 1997 Ontogenetic differences in retention of spatial learning tested with the Morris water maze. Dev Psychobiol 30:329-341

17. Mirmiran M, van den Dungen H, Uylings HB 1982 Sleep patterns during rearing under different environmental conditions in juvenile rats. Brain Res 233:287-298

18. Albin RL, Greenamyre JT 1992 Alternative excitotoxic hypotheses. Neurology 42:733-738

19. Beal MF 1995 Aging, energy, and oxidative stress in neurodegenerative diseases. Ann Neurol 38:357-366

20. Hoffman DJ, McGowan JE, Marro PJ, Mishra OP, Delivoria-Papadopoulos M 1994 Hypoxia-induced modification of the $N$-methyl-D-aspartate receptor in the brain of the newborn piglet. Neurosci Lett 167:156-160

21. Banasiak KJ, Haddad GG 1998 Hypoxia-induced apoptosis: effect of hypoxic severity and role of p53 in neuronal cell death. Brain Res 797:295-304

22. Decker MJ, Hue GEA, Feng PF, Rye DB 2001 Diminished wakefulness and locomotor hyperactivity in juvenile rats exposed to intermittent hypoxia as neonates. Proceedings of the Society for Neuroscience 31st Annual Meeting, San Diego, CA, p 27:A845847

23. Ali NJ, Pitson D, Stradling JR 1994 Natural history of snoring and related behaviour problems between the ages of 4 and 7 years. Arch Dis Child 71:74-76

24. Weissbluth M, Davis AT, Poncher J, Reiff J 1983 Signs of airway obstruction during sleep and behavioral, developmental, and academic problems. J Dev Behav Pediatr 4:119-121

25. Chervin RD, Archbold KH 2001 Hyperactivity and polysomnographic findings in children evaluated for sleep-disordered breathing. Sleep 24:313-320

26. Chervin RD, Dillon JE, Bassetti C, Ganoczy DA, Pituch KJ 1997 Symptoms of sleep disorders, inattention, and hyperactivity in children. Sleep 20:1185-1192

27. O'Brien LM, Holbrook CR, Klaus CJ, Bruner J, Raffield TJ, Rutherford J, Wang M, Mervis CB, Gozal D 2002 Sleep and neurocognitive function in 6-year old hyperactive children. Sleep 25:A55(abstr) 